\title{
Brain injury pathophysiology study by a multimodal approach in children with sickle cell anemia with no intra or extra cranial arteriopathy
}

Haematologica 2022

Volume 107(4):958-965

\author{
Valentine Brousse,$^{1,2,3,4}$ Corinne Pondarre ${ }^{5,6}$ Manoelle Kossorotoff, ${ }^{7}$ \\ Cecile Arnaud, ${ }^{5}$ Annie Kamdem, ${ }^{5}$ Mariane de Montalembert, ${ }^{1,2}$ \\ Benedicte Boutonnat-Faucher,${ }^{1}$ Slimane Allali, ${ }^{1,2,8}$ Hélène Bourdeau, ${ }^{9}$ \\ Keyne Charlot, ${ }^{10}$ Sebastien Bertil, ${ }^{11}$ Lydie da Costa, ${ }^{2,9,12,13}$ \\ Philippe Connes, ${ }^{2,14,15 \#}$ David Grévent ${ }^{16 \#}$ and Suzanne Verlhac ${ }^{17,18 \#}$
}

${ }^{1}$ AP-HP, Hôpital Necker Enfants Malades, Service de Pédiatrie Générale et Maladies infectieuses, Paris; ${ }^{2}$ LABEX GR-Ex, Paris; ${ }^{3}$ Institut National de la Transfusion Sanguine, UMR_S1134, INSERM, Paris; ${ }^{4}$ AP-HP, Hôpital Universitaire Robert Debré, ImmunoHématologie, Paris; ${ }^{5}$ Centre Intercommunal de Créteil, Service de Pédiatrie, Créteil; ${ }^{6}$ Paris XII University, INSERM U955, Créteil; ${ }^{7}$ AP-HP, Hôpital Necker Enfants Malades, Service de Neurologie Pédiatrique, Paris; ${ }^{8}$ Laboratory of Cellular and Molecular Mechanisms of Hematological Disorders and Therapeutical Implications, Paris Descartes - Sorbonne Paris Cité University, Imagine Institute, INSERM U1163, Paris; ${ }^{9}$ AP-HP, Hôpital Robert Debré, Service d'Hématologie Biologique, Paris; ${ }^{10}$ Unité de Physiologie des Exercices et Activités en Conditions Extrêmes, Département Environnements Opérationnels Institut de Recherche Biomédicale des Armées, Brétigny-sur-Orge; ${ }^{11}$ AP-HP, Service d'Hématologie Biologique, Hôpital Européen Georges Pompidou, Paris; ${ }^{12}$ Service d'Hématologie Biologique, Université de Paris, Paris; ${ }^{13}$ Hematim UR 4666, Amiens; ${ }^{14}$ Laboratoire LIBM EA7424, Equipe "Biologie Vasculaire et du Globule Rouge ", Université Claude Bernard Lyon 1, Villeurbanne; ${ }^{15}$ Institut Universitaire de France, Paris; ${ }^{16}$ AP-HP, Hôpital Necker Enfants Malades, Service d'Imagerie Pédiatrique, Paris; ${ }^{17}$ Centre Hospitalier Intercommunal de Créteil, Service d'Imagerie Médicale, Créteil and ${ }^{18}$ AP-HP, Hôpital Universitaire Robert Debré, Service d'Imagerie Médicale, Paris, France

${ }^{\#} P H, D G$ and SV contributed equally as co-senior authors

\section{Correspondence:}

VALENTINE BROUSSE

valentine.brousse@gmail.com

Received: December 21, 2020.

Accepted: April 14, 2021.

Pre-published: April 22, 2021.

https://doi.org/10.3324/haematol.2020.278226

(C)2022 Ferrata Storti Foundation

Material published in Haematologica is covered by copyright. All rights are reserved to the Ferrata Storti Foundation. Use of published material is allowed under the following terms and conditions:

https://creativecommons.org/licenses/by-nc/4.0/legalcode. Copies of published material are allowed for personal or internal use. Sharing published material for non-commercial purposes is subject to the following conditions:

https://creativecommons.org/licenses/by-nc/4.0/legalcode, sect. 3. Reproducing and sharing published material for commercial purposes is not allowed without permission in writing from the publisher.

\section{ABSTRACT}

$\mathrm{D}$ espite its high prevalence in children with sickle cell anemia (SCA), the pathophysiology of silent cerebral infarcts (SCI) remains elusive. The main objective of this study was to explore the respective roles of major determinants of brain perfusion in SCA children with no past or current history of intracranial or extracranial vasculopathy. We used a multimodal approach based notably on perfusion imaging arterial spin labeling (ASL) magnetic resonance imaging (MRI) and near infra-red spectroscopy (NIRS), as well as biomarkers reflecting blood rheology and endothelial activation. Out of 59 SCA patients (mean age $11.4 \pm 3.9$ yrs), eight (13\%) had a total of 12 SCI. Children with SCI had a distinctive profile characterized by decreased blood pressure, impaired blood rheology, increased P-selectin levels, and marked anemia. Although ASL perfusion and oximetry values did not differ between groups, comparison of biological and clinical parameters according to the level of perfusion categorized in terciles showed an independent association between high perfusion and increased sP-selectin, decreased red blood cell deformability, low hemoglobin F level, increased blood viscosity and no $\alpha$-thalassemia deletion. NIRS measurements did not yield additional novel results. Altogether, these findings argue for early MRI detection of SCI in children with no identified vasculopathy and suggest a potential role for ASL as an additional screening tool. Early treatment targeting hemolysis, anemia and endothelial dysfunction should reduce the risk of this under diagnosed and serious complication. 


\section{Introduction}

Brain injury is a major complication in sickle cell anemia (SCA) patients because of its high prevalence and devastating consequences. Historically, overt stroke used to be the most distressing clinical complication in childhood but its prevalence has dropped from $10 \%$ to $1-3 \%$ in high-income countries since the implementation of a preventive screening strategy by transcranial Doppler (TCD) coupled with chronic transfusion therapy in case of abnormal results. $1,2,3,4$ In sharp contrast, the contribution of silent cerebral infarcts (SCI) to global neurovascular burden has emerged, following the improved ability to detect subclinical lesions by magnetic resonance imaging (MRI). SCI are found in up to $37 \%$ of SCA children before the age of 14 years, can be detected as early as 1 year old and their prevalence increases with age. ${ }^{3,5,6}$ In addition, SCI are frequently associated with neurocognitive impairment. ${ }^{7,8}$ While overt ischemic stroke mainly occurs in the arterial territory of internal carotid or middle cerebral artery (usually with an associated stenosis or occlusion), silent infarcts tend to occur in the border zone areas of the brain, mainly in the deep white matter, including in patients without large vessel arteriopathy. This observation highly suggests that SCI may be related to alterations of perfusion and oxygenation. ${ }^{9}$ However, no clear pathophysiological process in SCI genesis has been demonstrated thus far, although anemia, both chronic and acute, seems to be a significant contributor. ${ }^{6}$ Conventional MRI shows brain injury in a pattern suggestive of hemodynamic compromise but it does not directly assess cerebral hemodynamics. By contrast, perfusion analysis by arterial spin labeling (ASL) allows to better evaluate the cerebral hemodynamic risk at a regional level, in a non-invasive manner, and may serve as a screening tool to identify children at risk of SCI.

Blood rheology is a key determinant of blood flow, vascular resistance and tissue perfusion. ${ }^{10}$ Increased blood viscosity in both adults and children with SCA has been associated with a risk of frequent vaso-occlusive crises. Moreover, SCA individuals with the greatest reduction in red blood cell (RBC) deformability would be at higher risk for developing leg ulcers, glomerulopathy and priapism. ${ }^{11,12,13}$ These studies also reported increased RBC aggregates strength in SCA patients with glomerulopathy or frequent priapism. Although a contribution of blood rheology has been speculated in overt stroke in SCA, ${ }^{14}$ there is no study focusing on SCI and blood rheology in this disease in the literature. Surprisingly, a study by Brown et al..$^{15}$ performed in patients with paraproteinemia or leukemia observed no association between cerebral blood flow and blood viscosity. Instead, the authors reported an inverse relationship between cerebral blood flow and arterial oxygen content, showing that regulatory mechanisms can maintain cerebral oxygen transport despite increased blood viscosity. The same negative association has been reported in children with SCA. ${ }^{16}$ However, the associations between brain oxygenation, cerebral blood flow in the different brain areas and blood rheology have never been investigated, and more particularly in the context of SCI. In order to better decipher the mechanisms involved in subclinical cerebral injury in children with SCA, we sought to explore the respective roles of hemolysis, abnormal blood rheology, cerebral oxygen supply and brain perfusion characteristics in children with no past or current history of intracranial or extracranial vasculopathy. We used a multimodal approach in a multi-centric pilot study where we i) compared several biomarkers (markers of endothelial injury and hemorheology), level of brain oxygenation (near infra-red spectroscopy [NIRS]) and perfusion imaging (TCD and arterial spin labeling [ASL] MRI) between children with newly discovered and without $\mathrm{SCI}$ and ii) tested the association between these parameters.

\section{Methods}

Consecutive patients from two French referral centers for sickle cell disease (University Hospital Necker-Enfants Malades and Centre Hospitalier Intercommunal de Creteil) were screened during routine visits according to the following inclusion criteria: i) SS or $\mathrm{S}-\beta^{\circ}$ thalassemia genotype; ii) no past history of abnormal or conditional transcranial and extracranial TCD; iii) at steady state (> 3 months from any vaso-occlusive event, transfusion or infection); iv) age between 5 and 17 years. The study was offered to all children meeting the inclusion criteria and regularly followed up since neonatal screening in the participating centers, between March 2015 and July 2016, with a target of 60 children (based on the expected number of SCA patients meeting the inclusion criteria in these centers and the pilot design of the study). Treatment by hydroxyurea was not an exclusion criterion, but chronic transfusion was. After written informed consent was obtained, a visit during which all investigations were performed was scheduled.

The protocol was approved by the ethics committee "Comite pour la Protection des Personnes Ile de France III" (014-A01575-42) and registered in clinicaltrials gov. Identifier: NCT 02909283.

Clinical parameters as well as relevant past medical events were collected and are available in the Online Supplementary Appendix. Biological parameters were measured for each patient: routine hematological and blood rheological parameters, and markers of endothelial activation (see the Online Supplementary Appendix).

TCD imaging (TCDI) was performed using a LOGIO E9 XDclear 2.0 ultrasound system (GE Healthcare, Milwaukee, WI, USA) at Necker - Enfants Malades and an Acuson S 2000 ultrasound system (Siemens Healthineers, Erlangen, Germany) at Centre Hospitalier Intercommunal de Creteil.

MR imaging was performed with a GE Signa HDxt 1.5-T system (GE Medical Systems, Milwaukee, WI, USA) and a 12-channel head-neck-spine coil in non-sedated children.

SCI were defined on MRI as hypersignals of cerebral parenchyma of at least $3 \mathrm{~mm}$ on T2 FLAIR sequence. Details on MRI sequences and image analysis including calculation of hypersignal volume are available in the Online Supplement Appendix.

Cerebral blood flow was measured using a three-dimensional pseudo-continuous arterial spin labeling (pCASL) sequence (repetition time msec/echo time msec, 4,428/10.5; postlabeling

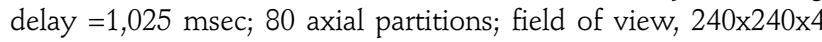
$\mathrm{mm}$; acquisition matrix, eight spiral arms in each three-dimensional partition with 512 points per arm; flip angle, $155^{\circ}$; acquisition time, 4 minutes 17 seconds).

Transcranial NIRS was performed using a dual-channel absolute cerebral oximeter FORE-SIGHT ${ }^{\circledR}$ (Branford, CT, USA).

\section{Statistics}

We first compared the different measured parameters in patients with and without SCI using an unpaired student $t$ test.

The cohort was divided into terciles of ASL values: hypo-perfusion (Low), normal perfusion (Middle) and hyper-perfusion (High) groups. the three groups were then compared using a one-way ANOVA with Tukey post hoc test. A $\chi^{2}$ test was used for the qualitative analyses. The significance level was defined as $P<0.05$ (SPSS, v. 20, IBM SPSS Statistics, Chicago, IL). 


\section{Results}

\section{General characteristics of the population}

Fifty-nine SCA patients (mean age $11.4 \pm 3.9$ yrs) at steady state were enrolled, of which $34(57.6 \%)$ were treated with hydroxyurea $(\mathrm{HU})$. Patients main clinical and biological characteristics are summarized in Table 1.

\section{Transcranial Doppler and magnetic resonance imaging analysis}

At the time of assessment, all TCD velocities were within normal ranges $(<170 \mathrm{~cm} / \mathrm{s})$ and there were no arte- rial abnormalities on MRA analysis. Twelve SCI (3-5 mm: $\mathrm{n}=8 ; 5-15 \mathrm{~mm}: \mathrm{n}=4,>15 \mathrm{~mm}: \mathrm{n}=0$ ) were observed in eight of $59(13 \%)$ children. The median volume of SCI was $41,57 \mathrm{~mm}$ (range, 15,8-362 mm). SCI were located in the right anterior border zone in six patients. Five patients had a single lesion, while three patients had $>1$ lesion, including two with bilateral lesions.

\section{Analysis of patients with silent cerebral infarcts}

Children with SCI had a lower past vaso-occlusive crisis (VOC) rate compared to children with no SCI (Table 1). Despite a comparable frequency of HU treatment in both

Table 1. General characteristics of the population.

\begin{tabular}{|c|c|c|c|c|}
\hline & All $(n=59)$ & $n 0-S C l(n=51)$ & SCI $(n=8)$ & $P$ \\
\hline $\operatorname{Sex}(M / F)$ & $20 / 39$ & $16 / 35$ & $4 / 4$ & 0.301 \\
\hline Age (yrs) & $11.4 \pm 3.9$ & $11.4 \pm 3.7$ & $11.6 \pm 4.9$ & 0.865 \\
\hline Ongoing HU ttt at inclusion ( $\mathrm{N} ; \%)$ & $33 ; 56$ & $30 ; 59$ & $3 ; 38$ & 0.259 \\
\hline Time since HU initiation (yrs) & $3.1 \pm 3.1$ & $3.2 \pm 3.2$ & $1.6 \pm 1.3$ & 0.170 \\
\hline Daily dose (mg/kg/day) mean (SD) & $23 \pm 4.6$ & $24.1 \pm 3.3$ & $12,8 \pm 0.5$ & 0.001 \\
\hline Age at beginning of HU initiation (yrs) & $8.3 \pm 4.3$ & $8.2 \pm 3.9$ & $9.0 \pm 5.4$ & 0.170 \\
\hline Alpha thalassemia (N; \%) & $24 ; 41$ & $23 ; 46$ & $1 ; 13$ & 0.074 \\
\hline G6PD deficiency (N; \%) & $4 ; 7$ & $4 ; 8$ & $0 ; 0$ & 0.412 \\
\hline VOC rate (events/yr) & $0.58 \pm 0.58$ & $0.65 \pm 0.60$ & $0.17 \pm 0.20$ & 0.001 \\
\hline ACS rate (events/yr) & $0.08 \pm 0.10$ & $0.09 \pm 0.10$ & $0.03 \pm 0.05$ & 0.157 \\
\hline Transfusion history (\%) & $23.7 \%$ & $23 \%$ & $25 \%$ & 0.96 \\
\hline Mean number of transfusions (n, SD) & $8(11)$ & $8(10)$ & $12(17)$ & 0.16 \\
\hline $\mathrm{SpO}_{2}(\%)$ & $99.0 \pm 1.1$ & $99.1 \pm 1.0$ & $98.6 \pm 2.0$ & 0.599 \\
\hline $\mathrm{DBP}(\mathrm{mmHg})$ & $68 \pm 11$ & $69 \pm 11$ & $61 \pm 7$ & 0.047 \\
\hline $\mathrm{SBP}(\mathrm{mmHg})$ & $110 \pm 11$ & $111 \pm 11$ & $106 \pm 10$ & 0.283 \\
\hline MAP (mmHg) & $82 \pm 9$ & $83 \pm 9$ & $76 \pm 8$ & 0.047 \\
\hline Pulse Pressure (mmHg) & $42 \pm 12$ & $42 \pm 12$ & $45 \pm 6$ & 0.437 \\
\hline $\mathrm{Hb}(\mathrm{g} / \mathrm{dL})$ & $8.8 \pm 1.2$ & $8.9 \pm 1.1$ & $8.2 \pm 1.1$ & 0.047 \\
\hline Hct (\%) & $25.0 \pm 3.3$ & $25.3 \pm 3.2$ & $23.0 \pm 3.0$ & 0.025 \\
\hline MCV (fl) & $82 \pm 12$ & $83 \pm 12$ & $77 \pm 10$ & 0.186 \\
\hline $\mathrm{MCHC}(\mathrm{g} / \mathrm{dL})$ & $35.0 \pm 1.5$ & $34.9 \pm 1.5$ & $35.8 \pm 0.9$ & 0.037 \\
\hline Reticulocyte count $\left(10^{9} / \mathrm{L}\right)$ & $225 \pm 102$ & $224 \pm 105$ & $229 \pm 88$ & 0.91 \\
\hline Platelets $\left(10^{9} / \mathrm{L}\right)$ & $343 \pm 154$ & $331 \pm 141$ & $418 \pm 220$ & 0.141 \\
\hline WBC count $\left(10^{9} / \mathrm{L}\right)$ & $8.98 \pm 3.61$ & $8.78 \pm 3.64$ & $10.29 \pm 3.36$ & 0.277 \\
\hline $\mathrm{HbF}(\%)$ & $16.0 \pm 10.1$ & $16.0 \pm 10.4$ & $16.1 \pm 8.2$ & 0.986 \\
\hline ASAT UI/L & $49 \pm 16$ & $48 \pm 16$ & $52 \pm 13$ & 0.548 \\
\hline LDH UI/L & $484 \pm 164$ & $485 \pm 173$ & $479 \pm 108$ & 0.938 \\
\hline Soluble E-selectin (ng/mL) & $73.4 \pm 29.6$ & $73.2 \pm 21.7$ & $74.5 \pm 39.7$ & 0.911 \\
\hline Soluble P-selectin (ng/mL) & $59.3 \pm 21.3$ & $57.1 \pm 20.7$ & $73.5 \pm 21.7$ & 0.047 \\
\hline CD34* cell $(/ \mathrm{mL})$ & $9185 \pm 6738$ & $9803 \pm 6773$ & $5788 \pm 6229$ & 0.301 \\
\hline RBC aggregation (\%) & $52 \pm 6$ & $52 \pm 7$ & $51 \pm 4$ & 0.811 \\
\hline RBC disaggregation threshold $\left(\mathrm{s}^{-1}\right)$ & $378 \pm 201$ & $359 \pm 191$ & $491 \pm 236$ & 0.037 \\
\hline RBC deformability (a.u.) & $0.49 \pm 0.09$ & $0.50 \pm 0.09$ & $0.44 \pm 0.07$ & 0.025 \\
\hline Blood viscosity (cP) & $5.35 \pm 0.93$ & $5.40 \pm 0.94$ & $5.04 \pm 0.83$ & 0.312 \\
\hline Hematocrit/blood viscosity (a.u.) & $4.72 \pm 0.59$ & $4.73 \pm 0.58$ & $4.62 \pm 0.69$ & 0.627 \\
\hline Right ctSO $2(\%)$ & $63 \pm 10$ & $63 \pm 10$ & $61 \pm 9$ & 0.662 \\
\hline Left $\mathrm{ctSO}_{2}(\%)$ & $63 \pm 10$ & $63 \pm 10$ & $64 \pm 9$ & 0.828 \\
\hline
\end{tabular}

M: male; F: female; SD: standard deviation; SCI: silent cerebral infarct; HU: hydroxyurea;VOC: vaso-occlusive crisis; ACS: acute chest syndrome; DBP: diastolic blood pressure; SBP systolic blood pressure; MAP: mean arterial pressure; MCHC: mean corpuscular hemoglobin concentration; MCV: mean corpuscular volume; Hct:hemtocrit; HbF: hemglobin F; ASAT: aspartate aminotransferase; LDH: lactate dehydrogenase; RBC: red blood cell; a.u.: artbitrary units; CP: centipoise; ctSO ${ }_{2}$ : cerebral tissue hemoglobin oxygen saturation; $P$-values are given for the comparison between the non-SCI and SCI groups. 
Table 2. Cerebral blood flow values in patients with or without silent cerebral infarct.

\begin{tabular}{lccc} 
& SCI $(\mathbf{n = 8})$ & \\
Right hemisphere perfusion (mL/100 g/min) & & & \\
ACA & $116.5 \pm 17.8(112.0-121.0)$ & $126.1 \pm 17.4(114.0-138.0)$ & 0.162 \\
Anterior Junctional & $122.9 \pm 21.1(117.0-129.0)$ & $123.8 \pm 16.2(113.0-135.0)$ & 0.909 \\
Superficial MCA & $111.9 \pm 17.9(107.0-117.0)$ & $116.5 \pm 13.8(107.0-126.0)$ & 0.489 \\
Caudate nucleus & $93.3 \pm 16.2(88.8-97.8)$ & $98.5 \pm 16.4(87.1-110.0)$ & 0.406 \\
Putamen & $82.5 \pm 14.2(78.6-86.4)$ & $90.0 \pm 13.5(80.7-99.3)$ & 0.168 \\
posterior Junctional & $120.6 \pm 23.3(114.0-127.0)$ & $120.5 \pm 16.9(109.0-132.0)$ & 0.988 \\
Left hemisphere perfusion (mL/100 g/min) & & & 0.117 \\
ACA & $116.5 \pm 19.4(111.0-122.0)$ & $132.0 \pm 23.9(115.0-149.0)$ & 0.887 \\
Anterior Junctional & $121.6 \pm 22.1(116.0-128.0)$ & $122.8 \pm 15.5(112.0-134.0)$ & 0.174 \\
Superficial MCA & $111.3 \pm 18.9(106.0-116.0)$ & $121.0 \pm 16.1(110.0-132.0)$ & 0.165 \\
Caudate nucleus & $94.3 \pm 16.0(89.9-98.7)$ & $102.8 \pm 14.2(93.0-113.0)$ & 0.058 \\
Putamen & $83.0 \pm 14.4(79.0-87.0)$ & $93.4 \pm 11.0(85.8-101.0)$ & 0.794 \\
Posterior Junctional & $119.9 \pm 26.4(113.0-127.0)$ & $122.5 \pm 24.3(106.0-139.0)$ & 0.295 \\
\hline Posterior fossa perfusion & & & \\
$\quad$ Right Cerebellar hemisphere & $88.1 \pm 8.2(85.8-90.3)$ & $84.1 \pm 8.2(78.4-89.8)$ & 0.277 \\
Left Cerebellar hemisphere & $89.6 \pm 16.9(85.0-94.2)$ & $85.4 \pm 8.1(79.8-91.0)$ & \\
\hline
\end{tabular}

No significant difference in cerebral blood flow values was evidenced between the two groups. Values are given as means \pm standard deviation (95\% Confidence Interval): SCI: silent cerebral infarcts; MCA: middle cerebral artery; ACA: anterior cerebral artery.

Table 3. Characteristics of the population according to the level of perfusion assessed by arterial spin labeling in the right anterior cerebral artery and categorized in terciles.

\begin{tabular}{|c|c|c|c|}
\hline & Lower CBF $(\mathrm{N}=20)$ & Middile CBF (N = 19) & Higher CBF $(\mathbb{N}=20)$ \\
\hline Age (yrs) & $12.4 \pm 4.2$ & $10.5 \pm 4.2$ & $11.2 \pm 3.2$ \\
\hline HU (\%) & 71 & 56 & 43 \\
\hline$\alpha$ thalassemia (\%) & 46 & 29 & $25^{*}$ \\
\hline G6PD deficiency (\%) & 31 & 35 & 35 \\
\hline VOC rate (events/yr) & $0.68 \pm 0.53$ & $0.47 \pm 0.43$ & $0.63 \pm 0.73$ \\
\hline ACS rate (events/yr) & $0.09 \pm 0.11$ & $0.06 \pm 0.08$ & $0.09 \pm 0.11$ \\
\hline $\mathrm{SpO}_{2}(\%)$ & $99.3 \pm 1.0$ & $99.1 \pm 1.2$ & $98.8 \pm 1.1$ \\
\hline DBP (mmHg) & $70 \pm 12$ & $67 \pm 11$ & $66 \pm 10$ \\
\hline $\mathrm{SBP}(\mathrm{mmHg})$ & $114 \pm 12$ & $108 \pm 12$ & $108 \pm 10$ \\
\hline MAP (mmHg) & $85 \pm 10$ & $81 \pm 9$ & $80 \pm 9$ \\
\hline $\mathrm{Hb}(\mathrm{g} / \mathrm{dL})$ & $9.2 \pm 1.2$ & $8.9 \pm 1.1$ & $8.3 \pm 1.0^{*}$ \\
\hline Hct $(\%)$ & $26.3 \pm 3.4$ & $25.2 \pm 3.3$ & $23.6 \pm 2.6^{*}$ \\
\hline MCV (fL) & $82 \pm 13$ & $81 \pm 11$ & $84 \pm 11$ \\
\hline $\mathrm{MCHC}(\mathrm{g} / \mathrm{dL})$ & $35.0 \pm 1.3$ & $35.2 \pm 1.0$ & $34.9 \pm 2.0$ \\
\hline Reticulocyte count $\left(10^{9} / \mathrm{L}\right)$ & $193 \pm 84$ & $229 \pm 112$ & $255 \pm 105^{*}$ \\
\hline Platelets $\left(10^{9} / \mathrm{L}\right)$ & $315 \pm 123$ & $314 \pm 141$ & $388 \pm 159$ \\
\hline White blood cells $\left(10^{9} / \mathrm{L}\right)$ & $7.99 \pm 3.37$ & $9.23 \pm 3.89$ & $9.91 \pm 3.51$ \\
\hline $\mathrm{HbF}(\%)$ & $19.4 \pm 11.3$ & $18.4 \pm 10.2$ & $11.9 \pm 6.5^{* \#}$ \\
\hline ASAT UI/L & $42 \pm 8$ & $50 \pm 15$ & $55 \pm 18^{*}$ \\
\hline LDH UI/L & $407 \pm 126$ & $515 \pm 154$ & $545 \pm 186$ \\
\hline $\mathrm{CRP} \mathrm{mg} / \mathrm{L}$ & $5.8 \pm 8.4$ & $6.0 \pm 5.0$ & $5.4 \pm 9.2$ \\
\hline E-selectin & $67.0 \pm 22.3$ & $68.5 \pm 35.0$ & $85.0 \pm 28.4$ \\
\hline P-selectin & $49.3 \pm 14.7$ & $58.2 \pm 21.3$ & $68.9 \pm 23.1^{* *}$ \\
\hline RBC aggregation (\%) & $52 \pm 6$ & $53 \pm 7$ & $50 \pm 5$ \\
\hline RBC disaggregation threshold $\left(\mathrm{s}^{-1}\right)$ & $390 \pm 222$ & $399 \pm 225$ & $356 \pm 174$ \\
\hline RBC deformability (a.u.) & $0.53 \pm 0.06$ & $0.51 \pm 0.09$ & $0.46 \pm 0.08^{*} \#$ \\
\hline Blood viscosity $(\mathrm{cP})$ & $5.75 \pm 1.03$ & $5.23 \pm 0.80$ & $5.18 \pm 0.93^{*}$ \\
\hline Hematocrit/blood viscosity (a.u.) & $4.66 \pm 0.70$ & $4.80 \pm 0.40$ & $4.67 \pm 0.67$ \\
\hline Right $\mathrm{ctSO}_{2}(\%)$ & $64 \pm 10$ & $62 \pm 9$ & $63 \pm 10$ \\
\hline Left ctSO $2(\%)$ & $64 \pm 13$ & $64 \pm 8$ & $64 \pm 10$ \\
\hline
\end{tabular}


groups, significant differences in biological profiles were evidenced: children with SCI had increased plasma P-selectin level and RBC disaggregation threshold, and lower hemoglobin ( $\mathrm{Hb}$ ) level and RBC deformability. Of note, daily dose of $\mathrm{HU}$ was significantly lower in patients with SCI compared to those with no SCI at the time of data collection but the sample size was too small ( $n=3$ of 8) to further interpret this finding.

In addition, diastolic blood pressure (DBP) and mean arterial pressure (MAP) were lower in patients with SCI. Oximetry data (right and left cerebral oxygenation levels) showed no significant difference between patients with and without SCI. Of note, there was no association between cerebral blood flow (CBF) values and the presence of SCI (Table 2).

\section{Arterial spin labeling perfusion imaging}

In the whole group of patients, CBF values showed no major asymmetry and correlated across all territories ( $\mathrm{r}$ ranging from 0.46 to $0.91 ; P$ ranging from $<0.01$ to $<0.001)$. Interestingly, comparison of patient subgroups according to $\mathrm{CBF}$ terciles in the arterial territories showed marked differences (see Table 3 for the right anterior cerebral artery territory, as an illustration). The group with
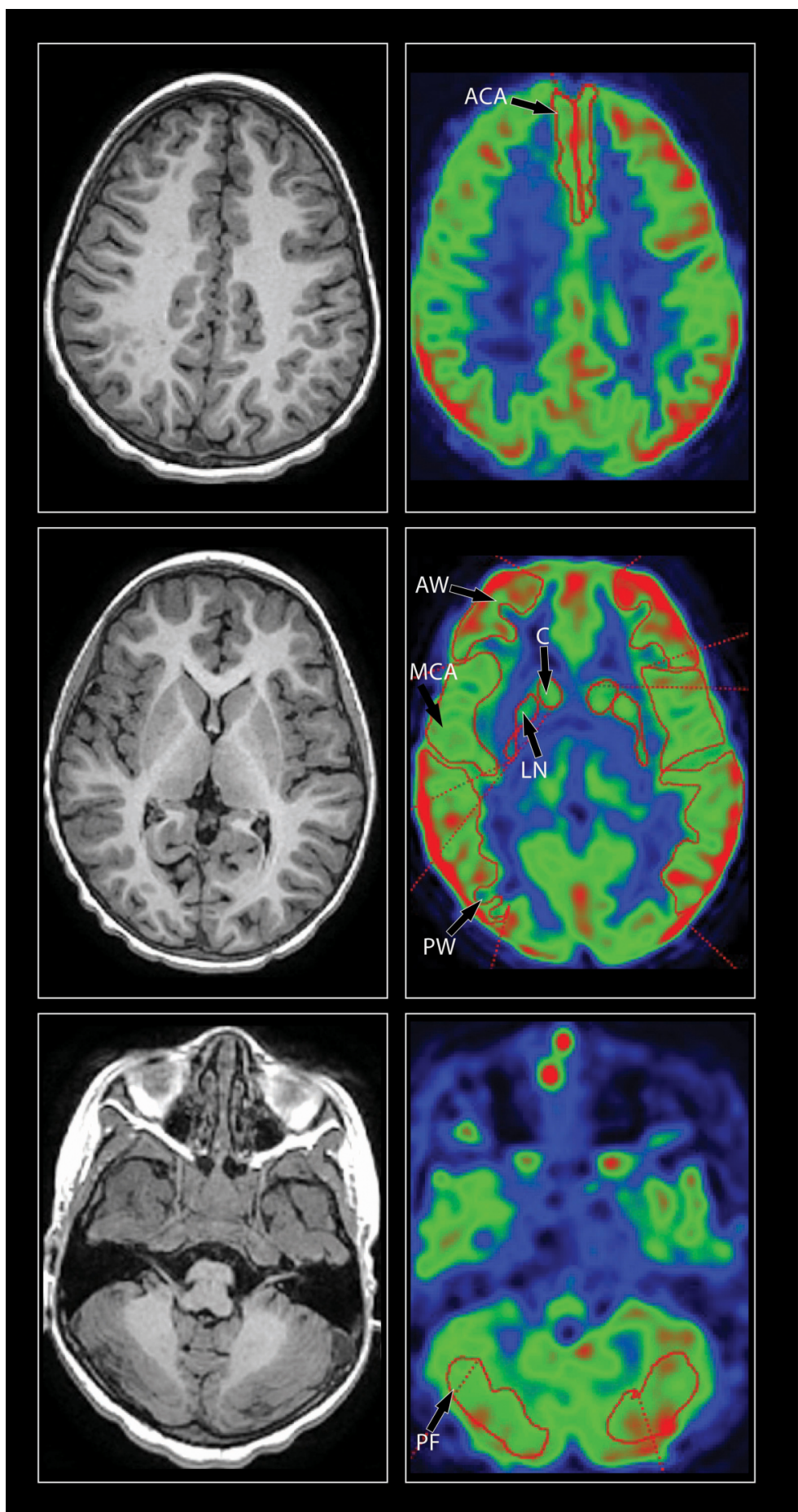

Figure 1. Regions of interest on the arterial spin labeling sequence. Left column: T1 weighted imaging. Right column: arterial spin labeling (ASL) imaging. First row: axial section tangential to the upper wall of the lateral ventricles, second row: axial section crossing the anterior and posterior white commissures (AC-PC), third row: axial section at the leve of the internal auditory canals. All axial images are parallel to CA-CP. ACA: anterior cerebral artery area; AW: anterior watershed area; MCA: middle cerebral artery area; PW: posterior watershed area; C: caudate nuclei; LN: lenticulostriate nuclei; PF: posterior fossa. 
higher CBF had increased markers of hemolytic anemia (lower $\mathrm{Hb}$, increased aspartate aminotransferase (ASAT), lactate dehydrogenase $[\mathrm{LDH}]$ and reticulocyte count), lower $\mathrm{HbF}$ level, increased marker of endothelial activation (sP-selectin value), lower RBC deformability and blood viscosity compared to the group with lower CBF. In addition, the frequency of patients with co inherited $\alpha$ thalassemia was lower. An ordinal multivariate analyses was performed to test the independent associations between the perfusion level (tercile groups) and the main parameters influencing blood flow. Blood viscosity was retained for the model because it is a key determinant of blood flow. ${ }^{10}$ Since blood viscosity is highly dependent on hematocrit/Hb and colinearity was indeed very strong between blood viscosity and hematocrit and $\mathrm{Hb}$ (variance inflation factor $[\mathrm{VIF}]=36.6$ and 37.4, respectively), hematocrit and $\mathrm{Hb}$ were not included in the multivariate model. Likewise, ASAT and reticulocyte count were not considered for the model as, like $\mathrm{LDH}$, they reflect hemolysis. In the multivariate model, which was highly $s$ i $g$ $n$ i $f$ i $c$ a $n \quad t$ $(P<0.001)$, all the parameters except $\mathrm{LDH}(\mathrm{P}=0.058)$, were independently associated with the level of perfusion (sP-selectin: $P<0.01$; RBC deformability: $P<0.01$; $\mathrm{HbF}$ level: $P<0.05$; blood viscosity: $P<0.05 ; \quad \alpha$-thalassemia: $P<0.05)$.

\section{Oximetry measurements}

In the whole population, cerebral oximetry results showed a significant positive correlation with $\mathrm{Hb}, \mathrm{SpO}_{2}$, $\mathrm{RBC}$ deformability, blood viscosity and $\mathrm{HbF}$ and a negative correlation with markers of hemolysis (total bilirubin and $\mathrm{LDH}$ ) as well as the RBC disaggregation threshold (Table 4). Of note, blood pressure, age, reticulocyte count, level of selectins and TCD velocities did not correlate with oximetry results. A multivariate analysis was performed for the right and left cerebral tissue hemoglobin oxygen saturation including $\mathrm{SpO}_{2}, \mathrm{Hb}, \mathrm{RBC}$ deformability, HbF, LDH and the RBC disaggregation threshold. $\mathrm{Hb}$ was preferred to blood viscosity as tissue oxygenation is thought to be highly dependent on the number of RBC and $\mathrm{Hb}$ concentration. None of the parameters was significant $\left(P=0.38\right.$ and $P=0.35$ for the right and left $\mathrm{ctSO}_{2}$, respectively), which may be explained by the fact that all are interrelated to affect brain oxygenation.

\section{Effect of hydroxyurea treatment}

$\mathrm{HU}$ treatment did not significantly impact the results of neurovascular explorations in this cohort, except for sEand sP-selectin levels that were significantly lower in children receiving treatment. Treated children received a daily dose of $\mathrm{HU}$ that was not a maximum tolerated dose but was a fairly high dose $(23+/-4.6 \mathrm{mg} / \mathrm{kg} /$ day $)$ and both groups had indeed comparable levels of $\mathrm{Hb}$ or $\mathrm{HbF}$. Although compliance was not specifically addressed, the decreased level of selectins in treated children argues for an effect of HU on alleviating endothelial injury, in addition to its known effect on hemolytic anemia. The crosssectional design of the study, however, does not allow to actually compare groups at baseline, i.e., before treatment initiation and precludes further interpretation. In line, the specific effect of HU in children with SCI (or the lack of, given the significantly low dose) is limited by the small sample size $(n=3)$.
Table 4. Correlations between cerebral oxygenation ( $\left.\mathrm{ctSO}_{2}\right)$ and biological parameters

\begin{tabular}{|c|c|c|}
\hline & eft ctSO2 $(\%)$ & Right ctSO2 (\%) \\
\hline Age (yrs) & $r=-0.12$ & $\mathrm{r}=-0.22$ \\
\hline $\mathrm{SpO}_{2}(\%)$ & $\mathrm{r}=0.35^{*}$ & $\mathrm{r}=0.43^{* *}$ \\
\hline $\mathrm{DBP}(\mathrm{mmHg})$ & $r=-0.08$ & $\mathrm{r}=0.14$ \\
\hline $\mathrm{SBP}(\mathrm{mmHg})$ & $r=-0.09$ & $r=-0.08$ \\
\hline MAP (mmHg) & $r=-0.09$ & $r=0.08$ \\
\hline $\mathrm{Hb}(\mathrm{g} / \mathrm{dL})$ & $\mathrm{r}=0.48^{* * *}$ & $\mathrm{r}=0.53^{* * *}$ \\
\hline Hct (\%) & $\mathrm{r}=0.53^{* * *}$ & $\mathrm{r}=0.56 * * *$ \\
\hline MCV (fL) & $r=0.07$ & $\mathrm{r}=0.17$ \\
\hline $\mathrm{MCHC}(\mathrm{g} / \mathrm{dL})$ & $r=-0.26$ & $r=-0.30^{*}$ \\
\hline Reticulocyte count $\left(10^{9} / \mathrm{L}\right)$ & $r=-0.06$ & $r=-0.12$ \\
\hline Platelets $\left(10^{9} / \mathrm{L}\right)$ & $r=-0.17$ & $r=-0.14$ \\
\hline White blood cells $\left(10^{9} \mathrm{~L}\right)$ & $r=-0.26$ & $r=-0.24$ \\
\hline $\mathrm{HbF}(\%)$ & $r=0.44^{* *}$ & $\mathrm{r}=0.45^{* *}$ \\
\hline ASAT UI/L & $r=-0.37 *$ & $r=-0.24$ \\
\hline LDH UI/L & $r=-0.51 * * *$ & $r=-0.43^{* *}$ \\
\hline CRP mg/L & $r=-0.07$ & $r=-0.24$ \\
\hline E-selectin & $r=-0.16$ & $r=-0.20$ \\
\hline P-selectin & $r=0.12$ & $r=0.07$ \\
\hline RBC aggregation (\%) & $\mathrm{r}=0.03$ & $r=-0.10$ \\
\hline RBC disaggregation threshold (s-1) & $r=-0.30^{*}$ & $r=-0.27^{*}$ \\
\hline RBC deformability (a.u.) & $\mathrm{r}=0.54^{* * *}$ & $\mathrm{r}=0.51^{* * *}$ \\
\hline Blood viscosity $(\mathrm{cP})$ & $r=0.30^{*}$ & $r=0.30^{*}$ \\
\hline Hematocrit/blood viscosity (a.u.) & $\mathrm{R}=0.21$ & $\mathrm{R}=0.17$ \\
\hline
\end{tabular}

DBP: diastolic blood pressure; SBP: systolic blood pressure; Hb: hemoglobin; Hct: hemtocrit; MCV: mean corpuscular volume; MCHC: mean corpuscular hemoglobin concentration; ASAT: aspartate aminotransferase; $\mathrm{LDH}$ : lactate dehydrogenase; CRP: C-reactive protein; a.u.: arbitrary units; RBC: red blood cell; $\mathrm{ctSO}_{2}$ : cerebral tissue hemoglobin oxygen saturation Significant correlations: ${ }^{*} P<0.05 ;{ }^{* *} P<0.01 ;{ }^{*}{ }^{*} P<$ 0.001 .

\section{Discussion}

In this study of 59 young children highly selected for no present or past history of cerebral and extra cranial vasculopathy, a prevalence of eight of $59(13 \%)$ children with SCI was found, illustrating the residual burden of neurovascular injury in patients having received recommended follow-up. SCI predominated in the fronto-parietal white matter at the junction between the anterior and the middle cerebral artery territories, as previously described. ${ }^{17}$ Expectedly, on T2 FLAIR sequence, no SCI $>15 \mathrm{~mm}$ were found which would have presumably translated in clinically detectable symptoms. Likewise, volumes of SCI were within previously described ranges. ${ }^{18}$ Consistent with the inclusion criteria of no past conditional or abnormal TCD or transient vasculopathy, there was no arterial abnormality on MRA analysis. SCI and more specifically their size, volume and localization may impact cognitive function. In this study cognitive testing was not performed and subsequently cognitive consequences related to SCI cannot be inferred. Nevertheless, the frequency of SCI in these highly selected children plead for early identification of silent lesions in order to further explore cognitive functions for early implementation of supportive learning skills. In line with recent recommendations, MRI screening, is therefore highly recommended in all children with SCA, including in those with no identified vasculopathy. ${ }^{19}$ 
Given the absence of large vessel vasculopathy in these children, SCI are presumably unrelated to ischemia occurring downstream of a large vessel stenosis. SCI may nevertheless share common risk factors with large vessel vasculopathy and result from impaired perfusion. Indeed, we found distinctive clinical and biological features in children with SCI. In line with previous reports, a markedly increased hemolytic and anemic profile favoring SCI was evidenced, consistent with a lower pain rate. ${ }^{20,21} \mathrm{HU}$ treatment did not significantly impact these data, but interpretation is very limited given the small sample size of treated children with SCI ( $n=3)$, and the low daily dose at the time of data collection. Interestingly, despite significantly greater anemia in children with SCI, blood viscosity did not significantly differ across groups because RBC deformability was reduced in the former group, which exerts opposite effects on blood viscosity. ${ }^{22}$ Intravascular hemolysis is a major determinant of vascular and endothelial dysfunction. ${ }^{23}$ Consistent with this, an increased level of sP-selectin, a marker of endothelial activation, was evidenced and was associated with SCI. sP-selectin could potentially serve as a biomarker of cerebral injury in children. ${ }^{6}$ In contrast with previous studies, ${ }^{24}$ a lower diastolic and mean arterial pressure was found in children with SCI. Altogether, this data strengthens the hemodynamic pathophysiology of SCI, whereby a further drop in pressure and/or blood flow in children with severe anemia results in ischemia in the border zone region, characterized by terminal arterial supply. Furthermore, hemorheological exploration of these patients suggests that increased RBC aggregate strength and decreased red cell deformability may also influence the risk of SCI. Deformable RBC mostly flow in single file in capillaries and $\mathrm{RBC}$ aggregates need to be fully dispersed before entering into the microcirculation. ${ }^{22,10}$ Consequently, decreased RBC deformability and increased RBC aggregates strength may both impair blood flow at the entry of small capillaries and affect tissue perfusion.

Cerebral blood flow begins at a low level in the perinatal period, increases to a peak value at 3-8 years of age and then gradually decreases to adult levels with a negative correlation with age. ${ }^{25}$ Several reports have documented elevated CBF in the grey matter of children with SCA, compared to normal controls, ${ }^{26-28}$ a finding attributed to the compensatory increase in blood velocity and flow secondary to baseline anemia. This study allowed further insight by showing that, regardless of concomitant HU treatment, the level of perfusion was independently associated with blood viscosity (which is negatively correlated to anemia), $\mathrm{HbF}$ level, endothelial activation, $\alpha$-thalassemia status, and marginally associated with the level of hemolysis. ASL perfusion may therefore serve as an additional screening tool that integrates all these parameters to identify children at risk of subclinical injury, beyond the known risk factor, and regardless of cerebral vasculopathy. Thresholds for risk assessment will need to be determined by further prospective studies.

Oximetry analysis yielded expected and coherent results. NIRS is a non-invasive measurement of cerebral oxygenation, that varies with $\mathrm{SpO}_{2}$ and $\mathrm{Hb}$. In line with previous reports, we show that cerebral oxygenation is low in SCA patients. ${ }^{29,30,31}$ Our results also confirmed an association between RBC rheological parameters and cerebral oxygenation, suggesting that patients with the most deformable $\mathrm{RBC}$ and less robust $\mathrm{RBC}$ aggregates would have increased cerebral perfusion and oxygenation. However, because NIRS is unable to measure cerebral oxygenation at a depth of interest and is limited to the anterior territories, its additional predictive value remains to be demonstrated, particularly in patients at risk of white matter SCI.

This study has a number of limitations. Its cross-sectional design does not allow associations to be interpreted as causalities and its sample size was small, particularly regarding the number of patients with SCI treated by HU. It is possible that the lack of association between elevated CBF values and the presence of SCI was due to the low power of the study, for instance. Regarding imaging techniques, ASL MRI has also inherent limitations. In particular the transit time is reduced in SCA patients relative to non-anemic children. Thus, the labeling efficiency and postlabeling delay may not be adequate in every patient and may modify the perfusion signal measured by ASL. ${ }^{28}$ Another parameter, the fixed value of the T1-longitudinal relaxation time of blood used for $\mathrm{CBF}$ quantification may not be adequate in every individual patient, given its dependence on hematocrit and blood composition. Another limitation is that CBF measurements were made in grey matter, while silent infarcts are located preferentially in white matter. At a magnetic field strength of 1.5 Tesla, the signal-to-noise ratio of the resulting perfusion map is too low in the cerebral white matter to allow accurate measurements. Despite these limitations, however, our ASL results adequately reflected the level of perfusion in different territories of the brain. In fact, our results were consistent with previous reports regarding the influence of both anemia and age and further allowed novel coherent results regarding the influence of endothelial activation and blood rheology for instance, and more generally the possible pathophysiology of SCI genesis.

Altogether the findings of this study suggest that SCI may, like overt stroke, preferably occur in otherwise paucisymptomatic children with marked hemolytic anemia and endothelial dysfunction. Cerebral blood flow measurements by ASL MRI may help assess the quality of perfusion at a microvascular level in children with no vasculopathy but nevertheless at risk of subclinical injury. An early disease-modifying treatment like HU, which improves all afore-mentioned factors associated with SCI may therefore decrease SCI risk as well, in addition to its known beneficial effect on TCD velocities and stroke risk. ${ }^{27,32,33}$ Decreasing hemolytic anemia, improving RBC rheological characteristics and limiting endothelial injury will help avoid cerebral injury, particularly in case of further aggression such as arterial stenosis, acute hypoxia, drop in $\mathrm{Hb}$ or increased metabolic demand. Large prospective trials evaluating the protective effect of HU on SCI are ongoing and will hopefully confirm such beneficial effect. It is possible that new therapeutic approaches such as P-selectin blockade by monoclonal or pan antibodies, may have further protective effects in this particular complication.

\section{Disclosures}

$V B, C P$ and $M d M$ report honoraria and expert/consultancy testimony for Addmedica.

\section{Contributions}

$V B, M K, D G$ and $S V$ designed the study; VB, CP, CA, AK, $M d M, B B F$ and $S A$ enrolled patients; $H B$ and $L d C$ were responsible for biological data, except for $E$ - and P-selectins that were measured by $S B ; S V$ and $D G$ were responsible for imaging 
data; KC and $P C$ were responsible for oximetry and hemorheological data interpretation; $V B, P C, C P, M K, D G$, and $S V$ analyzed the data; $P C$ was responsible for statistical analysis; VB and $P C$ wrote the manuscript. All authors reviewed, edited and approved the manuscript.

\section{Acknowledgments}

We wish to thank Wiam BHIA, Nicholas Renaud, Elisabeth
Hullier-Amar and Isabelle Buffet from the Fondation Institut Imagine.

\section{Funding}

This research was funded by $L A B E X-G R-E x$, grant number GR-EX14/ParisDiderot/ and an additional grant was obtained from AddMeddica.

\section{References}

1. Ohene-Frempong K, Weiner SJ, Sleeper LA, et al. Cerebrovascular accidents in sickle cell disease: rates and risk factors. Blood. 1998;91(1):288-294.

2. Brousse V, Arnaud C, Lesprit E, et al. Evaluation of outcomes and quality of care in children with sickle cell disease diagnosed by newborn screening: a real-world nation-wide study in France. J Clin Med. 2019;8(10):1594

3. Bernaudin F, Verlhac S, Arnaud C, et al. Impact of early transcranial Doppler screening and intensive therapy on cerebral vasculopathy outcome in a newborn sickle cell anemia cohort. Blood. 2011;117(4): 1130-1140.

4. Adams RJ, McKie VC, Hsu L, et al. Prevention of a first stroke by transfusions in children with sickle cell anemia and abnormal results on transcranial Doppler ultrasonography. N Engl J Med. 1998; 339(1):5-11.

5. McCarville MB, Goodin GS, Fortner G, et al. Evaluation of a comprehensive transcranial doppler screening program for children with sickle cell anemia. Pediatr Blood Cancer. 2008;50(4):818-821.

6. DeBaun MR, Kirkham FJ. Central nervous system complications and management in sickle cell disease. Blood. 2016;127(7):829838.

7. Bernaudin F, Verlhac S, Freard F, et al. Multicenter prospective study of children with sickle cell disease: radiographic and psychometric correlation. J Child Neurol. 2000;15(5):333-343.

8. DeBaun MR, Schatz J, Siegel MJ, et al. Cognitive screening examinations for silent cerebral infarcts in sickle cell disease. Neurology. 1998;50(6):1678-1682.

9. Fields ME, Guilliams KP, Ragan DK, et al. Regional oxygen extraction predicts border zone vulnerability to stroke in sickle cell disease. Neurology. 2018;90(13):e1134e1142.

10. Nader E, Skinner S, Romana M, et al. Blood rheology: key parameters, impact on blood flow, role in sickle cell disease and effects of exercise. Front Physiol. 2019;10:1329.

11. Lamarre Y, Romana M, Lemonne N, et al. Alpha thalassemia protects sickle cell anemia patients from macro-albuminuria through its effects on red blood cell rheological properties. Clin Hemorheol Microcirc. 2014;57(1):63-72.
12. Connes P, Lamarre Y, Hardy-Dessources $M-D$, et al. Decreased hematocrit-to-viscosity ratio and increased lactate dehydrogenase level in patients with sickle cell anemia and recurrent leg ulcers. PLoS One. 2013;8(11):e79680.

13. Cita K-C, Brureau L, Lemonne N, et al. Men with sickle cell anemia and priapism exhibit increased hemolytic rate, decreased red blood cell deformability and increased red blood cell aggregate strength. PLoS One. 2016;11(5):e0154866.

14. Connes P, Verlhac S, Bernaudin F. Advances in understanding the pathogenesis of cerebrovascular vasculopathy in sickle cell anaemia. Br J Haematol. 2013;161(4):484498.

15. Brown MM, Marshall J. Regulation of cerebral blood flow in response to changes in blood viscosity. Lancet. 1985;1(8429):604609.

16. Bush AM, Borzage MT, Choi S, et al. Determinants of resting cerebral blood flow in sickle cell disease. Am J Hematol. 2016;91(9):912-917.

17. Ford AL, Ragan DK, Fellah S, et al. Silent infarcts in sickle cell disease occur in the border zone region and are associated with low cerebral blood flow. Blood. 2018; 132(16):1714-1723

18. van der Land V, Hijmans CT, de Ruiter M, et al. Volume of white matter hyperintensities is an independent predictor of intelligence quotient and processing speed in children with sickle cell disease. $\mathrm{Br} \mathrm{J}$ Haematol. 2015;168(4):553-556.

19. DeBaun MR, Jordan LC, King AA, et al. American Society of Hematology 2020 guidelines for sickle cell disease: prevention, diagnosis, and treatment of cerebrovascular disease in children and adults. Blood Adv. 2020;4(8):1554-1588.

20. Bernaudin F, Verlhac S, Arnaud C, et al. Chronic and acute anemia and extracranial internal carotid stenosis are risk factors for silent cerebral infarcts in sickle cell anemia. Blood. 2015;125(10):1653-1661.

21. Kinney TR, Sleeper LA, Wang WC, et al. Silent cerebral infarcts in sickle cell anemia: a risk factor analysis. The Cooperative Study of Sickle Cell Disease. Pediatrics. 1999;103(3):640-645.

22. Baskurt OK, Meiselman HJ. Blood rheology and hemodynamics. Semin Thromb Hemost. 2003;29(5):435-450.

23. Sundd P, Gladwin MT, Novelli EM Pathophysiology of sickle cell disease.
Annu Rev Pathol. 2019;14:263-292.

24. DeBaun MR, Sarnaik SA, Rodeghier MJ, et al. Associated risk factors for silent cerebral infarcts in sickle cell anemia: low baseline hemoglobin, sex, and relative high systolic blood pressure. Blood. 2012;119(16):36843690.

25. Petcharunpaisan S, Ramalho J, Castillo M Arterial spin labeling in neuroimaging World J Radiol. 2010;2(10):384-398.

26. Oguz KK, Golay X, Pizzini FB, et al. Sickle cell disease: continuous arterial spin-labeling perfusion $M R$ imaging in children. Radiology. 2003;227(2):567-574.

27. Helton KJ, Paydar A, Glass J, et al. Arterial spin-labeled perfusion combined with segmentation techniques to evaluate cerebra blood flow in white and gray matter of children with sickle cell anemia. Pediatr Blood Cancer. 2009;52(1):85-91.

28. Gevers S, Nederveen AJ, Fijnvandraat K, et al. Arterial spin labeling measurement of cerebral perfusion in children with sickle cell disease. J Magn Reson Imaging. 2012; 35(4):779-787.

29. Waltz X, Pichon A, Mougenel D, et al. Hemorheological alterations, decreased cerebral microvascular oxygenation and cerebral vasomotion compensation in sickle cell patients. Am J Hematol. 2012; 87(12):1070-1073

30. Nahavandi M, Nichols JP, Hassan M Gandjbakhche A, Kato GJ. Near-infrared spectra absorbance of blood from sickle cell patients and normal individuals. Hematology. 2009;14(1):46-48.

31. Charlot K, Antoine-Jonville S, Moeckesch $\mathrm{B}$, et al. Cerebral and muscle microvascular oxygenation in children with sickle cell disease: Influence of hematology, hemorheology and vasomotion. Blood Cells Mol Dis. 2017;65:23-28.

32. Ware RE, Davis BR, Schultz WH, et al Hydroxycarbamide versus chronic transfusion for maintenance of transcranial doppler flow velocities in children with sickle cell anaemia-TCD with transfusions changing to hydroxyurea (TWiTCH): a multicentre, open-label, phase 3 , non-inferiority trial. Lancet. 2016;387(10019):661670.

33. Bernaudin F, Verlhac S, Peffault de Latour R, et al. Association of matched sibling donor hematopoietic stem cell transplantation with transcranial Doppler velocities in children with sickle cell anemia. JAMA. 2019; 321(3):266-276 